\title{
Journal of Digital Imaging
}

VOL 13, NO 4

NOVEMBER 2000

\section{Computers in Imaging and Health Care: Now and in the Future}

\author{
Ronald L. Arenson, Katherine P. Andriole, David E. Avrin, and Robert G. Gould
}

Early picture archiving and communication systems (PACS) were characterized by the use of very expensive hardware devices, cumbersome display stations, duplication of database content, lack of interfaces to other clinical information systems, and immaturity in their understanding of the folder manager concepts and workflow reengineering. They were implemented historically at large academic medical centers by biomedical engineers and imaging informaticists. PACS were nonstandard, home-grown projects with mixed clinical acceptance. However, they clearly showed the great potential for PACS and filmless medical imaging. Filmless radiology is a reality today. The advent of efficient softcopy display of images provides a means for dealing with the ever-increasing number of studies and number of images per study. Computer power has increased, and archival storage cost has decreased to the extent that the economics of PACS is justifiable with respect to film. Network bandwidths have increased to allow large studies of many megabytes to arrive at display stations within seconds of examination completion. PACS vendors have recognized the need for efficient workflow and have built systems with intelligence in the management of patient data. Close integration with the hospital information system (HIS)-radiology information system (RIS) is critical for system functionality. Successful implementation of PACS requires integration or interoperation with hospital and radiology information systems. Besides the economic advantages, secure rapid access to all clinical information on patients, including imaging studies, anytime and anywhere, enhances the quality of patient care, although it is difficult to quantify. Medical image management systems are maturing, providing access outside of the radiology department to images and clinical information throughout the hospital or the enterprise via the Internet. Small and medium-sized community hospitals, private practices, and outpatient centers in rural areas will begin realizing the benefits of PACS already realized by the large tertiary care academic medical centers and research institutions. Hand-held devices and the Worldwide Web are going to change the way people communicate and do business. The impact on health care will be huge, including radiology. Computer-aided diagnosis, decision support tools, virtual imaging, and guidance systems will transform our practice as value-added applications utilizing the technologies pushed by PACS development efforts.
Outcomes data and the electronic medical record (EMR) will drive our interactions with referring physicians and we expect the radiologist to become the informaticist, a new version of the medical management consultant.

Copyright $\odot 2000$ by W.B. Saunders Company

KEY WORDS: picture archiving and communication systems (PACS), image storage and retrieval, folder manager, workflow manager, radiology information systems, computers, digital radiology.

\section{EARLY USES OF COMPUTERS IN RADIOLOGY}

C OMPUTERS have been used in radiology for many years, with the earliest applications focused on administrative functions such as billing and patient registration. Radiology information systems (RIS) developed during the 1970s at academic centers with commercial systems emerging in the early 1980s. Nuclear medicine was one of the first disciplines to use computers for images, followed by interventional radiologists use of digital subtraction angiography (DSA). Computerized tomography (CT, 1970s) and magnetic resonance imaging (MRI, 1980s), of course, depend on computers for the creation of the images from the data collected. Picture archiving and communication systems (PACS) began to emerge in the 1980s, but commercial systems lagged until the 1990s.

Most departments of radiology now depend on a RIS for such functions as registration, scheduling, film library management, patient tracking, results reporting, management reporting, and billing. These

From the Department of Radiology, University of California San Francisco, San Francisco, CA.

Address reprint requests to Ronald $L$. Arenson, $M D$, University of California, San Francisco, Department of Radiology, 505 Parnassus Ave, M-391, San Francisco, CA 94143-0628.

Copyright (C) 2000 by W.B. Saunders Company

0897-1889/00/1304-0005\$10.00/0

doi:10.1053/jdim.2000.17803 
modules have evolved over the years and are now quite powerful and sophisticated. As will be presented below, the boundaries between the RIS, the PACS, and the hospital information system (HIS) are becoming blurred as integration and interfacing of these separate systems improves. In addition, the Worldwide Web is going to fundamentally change the way in which we communicate and interact with these systems on which we depend so heavily.

\section{HISTORICAL PACS}

Picture archiving and communication systems (PACS) or medical image management systems, as they are sometimes called, are a collection of technologies used to carry out digital medical imaging. PACS are used to digitally acquire medical images from the various modalities such as CT, MRI, ultrasound (US), nuclear medicine (NM), and digital projection radiography. The image data and pertinent information are transmitted to other and possibly remote locations over networks, where they can be displayed on computer workstations for soft copy viewing in multiple locations simultaneously. Data are secured and archived on digital media such as optical disks or tape, and can be automatically retrieved as necessary.

Research into the design and implementation of PACS began over 10 years ago as a hardware problem, testing the limitations of existing computer engines, display monitors, network technologies, and storage devices. The first implementations were largely initiated in radiology departments and biomedical engineering or informatics laboratories at academic medical centers. PACS tended to be developed in-house (or home grown), and were therefore one-of-a-kind systems. ${ }^{1}$

Early PACS were characterized by the use of costly Unix mainframe or minicomputer workstation servers and display stations. They used very expensive high spatial resolution, low brightness gray-scale display monitors, and high-priced storage devices. Display station graphical user interfaces were cumbersome and rather nonintuitive. PACS databases duplicated large amounts of relevant data already contained in other medical information systems because they were isolated, self-contained systems with poor or nonexistent interfacing to other data stores.

Due to the lack of interfacing to radiology or hospital information systems, the image data man- agement systems had poor implementation of workflow or folder manager concepts, which describe how appropriate data are delivered where and when. In the film-based diagnostic imaging department, folder manager rules such as pulling relevant prior imaging examinations (prefetching), delivery of data to anticipated locations of need (preemptive autorouting), standardized viewing (hanging protocols), and film filing (hierarchical storage management algorithms), are functions carried out by $\mathrm{x}$-ray technologists, film file room clerks, and other experienced clinical personnel. ${ }^{1-4}$ In PACS, these functions need to be automatically performed by computers.

Deficits in the understanding and incorporation of folder manager routines, as well as workflow reengineering, resulted in mixed clinical acceptance and use of early PACS. Although digital data were being acquired into these early systems, PACS failed to eliminate the use of film. Image presentation was suboptimal, and data retrieval was difficult because PACS databases were not patient-centric, but rather examination- or imagecentric. Information tools must be provided to radiologist and clinician end-users, enabling them to negotiate the healthcare environment using patient-centric thinking.

\section{MEDICAL IMAGING TODAY WITH PACS}

Many medium and large departments of radiology have now successfully implemented PACS. A number of these departments use mini-PACS, systems installed to solve particular problems in parts of the department, but not the whole department. Many of these departments still create film for archival purposes, if not also for interpretation by the radiologists. The elimination of film and the processes to manage film are necessary to achieve the greatest cost reductions and efficiencies with PACS. Yet, only a few departments have achieved a filmless environment for a number of important reasons discussed in the next section.

Acquisition and display of the inherently digital cross-sectional modalities such as CT, MR, US, and NM have proven more easily implementable in filmless fashion and the first to achieve clinical acceptance. More efficient operations and cost effectiveness have been attainable within radiology departments with PACS, but health care enterprisewide delivery of imaging information remains problematic. This has resulted in many function- 
ally filmless diagnostic imaging departments continuing to print film for the bulk of their examinations.

Digital projection radiography has proven more difficult to cost justify for medium to low volume practices. Because this type of imaging typically accounts for the largest percentage of studies performed at most health care institutions, reductions in film printing have only been realized in highvolume environments. Computed radiography (CR), or imaging with photostimulable phosphors, has been implemented successfully for portable or bedside examinations in intensive care or emergency department settings with some digital radiography (DR) being used experimentally for mammography and clinically replacing upright chest and table Bucky trays. ${ }^{5}$

Even as the industry has entered the medical imaging delivery market, many PACS have continued to be self-supported and maintained. Most of the initial PACS vendors were film companies and imaging modality manufacturers. Computer, database, Internet, and information management companies are beginning to offer PACS products. The industry appears to be heading toward PACS service providers, through which vendors install, support, and maintain clinical image and information systems.

It is envisioned that PACS can provide a costeffective mechanism for universal delivery of highquality diagnostic radiology in today's healthcare environment. The potential impact of the use of PACS include more expedient care, more efficient workflow, and better quality of medical imaging services through image manipulation and information delivery. PACS today are the result of the convergence of technologic and economic factors.

\section{ENABLING TECHNOLOGIES FOR PACS AND FILMLESS RADIOLOGY}

Technology had to catch up to the concepts of PACS. Specifically, developments had to occur in at least 5 broad areas: standards, computer speed, network speed, archival devices, and recognition of the importance of image management based on integration with the radiology and hospital clinical information systems.

\section{Standards}

The most important and necessary standard is DICOM (digital imaging and communications in medicine) that began as a joint effort between the American College of Radiology (ACR) and the National Electronic Manufacturers Association (NEMA). The original standard for digital images defined an image file format, included hardware requirements for physical connections, and defined a communication protocol. ${ }^{6}$ However, major manufacturers in large part never implemented the initial standard because it allowed for only pointto-point image transfers, there was no real demand for the ACR/NEMA standard by the users, and the manufacturers had proprietary concerns. The DICOM standard currently implemented is the result of more than 10 years of development. It continues to evolve as new technology, for example, new storage devices and new imaging techniques develop, such as 4-dimensional image data sets (including the time variable), unification of soft copy display, and compression. DICOM defines a network protocol that allows devices on the network to negotiate services to be performed (eg, store, query, retrieve, print). Manufacturers must provide a "conformance statement" that describes how DICOM has been implemented for a given device and what services the device supports.

The use of DICOM externally is a critical component of a PACS architecture. For various reasons such as achieving better image transfer efficiency many modalities and PACS do not maintain strict DICOM representation internally for storage or communication. The multimodality, multivendor, nature of any radiology department requires integration, which is achievable only through a robust, dynamic open communication standard.

A second standard that has been required is HL7. ' It permits patient demographics and patient information such as admission, discharge, transfer (ADT) and scheduled imaging procedures to be known by the RIS and PACS. This standard is more of a framework for interfaces than a welldefined, structured standard like DICOM. In HL7 there is no defined data object, data elements are less well defined, and of undefined length. However, this standard has permitted the development of more efficient PACS, allowing historical image data to be moved based on anticipated procedures. An additional benefit of HIS-RIS and PACS communication is the ability to verify and validate the integrity of images coming into the PACS to assure that all images are handled correctly. 
Table 1. Bandwidth of Commonly Used Communication Methods

\begin{tabular}{|c|c|c|c|}
\hline & \multirow{2}{*}{$\begin{array}{l}\text { Maximum } \\
\text { Bandwidth }\end{array}$} & \multicolumn{2}{|c|}{$\begin{array}{c}\text { Minimum Transmission Time } \\
(\mathrm{sec})\end{array}$} \\
\hline & & 1 Mbyte & 80 Mbytes \\
\hline Modem & $\begin{array}{l}56 \text { Kbits/ } \\
\text { sec }\end{array}$ & $\begin{array}{l}142.8 \\
(2.4 \mathrm{~min})\end{array}$ & $\begin{array}{l}11,489 \\
(190 \mathrm{~min})\end{array}$ \\
\hline ISDN & $\begin{array}{l}128 \text { Kbits/ } \\
\text { sec }\end{array}$ & 62.5 & $\begin{array}{l}5,000 \\
\quad(83.3 \mathrm{~min})\end{array}$ \\
\hline DSL & $\begin{array}{l}384 \text { Kbits/ } \\
\text { sec }\end{array}$ & 20.8 & $\begin{array}{l}1667 \\
(27.8 \mathrm{~min})\end{array}$ \\
\hline Ethernet & $\begin{array}{l}10 \mathrm{Mbits} / \\
\mathrm{sec}\end{array}$ & 0.8 & 64 \\
\hline Fast Ethernet & $\begin{array}{l}100 \text { Mbits/ } \\
\text { sec }\end{array}$ & 0.08 & 6.4 \\
\hline Gigabit Ethernet & $\begin{array}{l}1000 \text { Mbits/ } \\
\text { sec }\end{array}$ & 0.008 & 0.64 \\
\hline ATM & $\begin{array}{l}155 \text { Mbits/ } \\
\text { sec }\end{array}$ & 0.052 & 4.1 \\
\hline $\begin{array}{l}\text { Fast Ethernet w/10:1 } \\
\text { compression }\end{array}$ & $\begin{array}{l}100 \text { Mbits/ } \\
\text { sec }\end{array}$ & 0.008 & 0.64 \\
\hline
\end{tabular}

\section{Speed and Bandwidth}

The content of a digital image is a function of the amount of digital data within the image. Higher spatial resolution for a given image area requires more pixels. Greater contrast sensitivity and increased dynamic range require greater pixel depth. Filmless radiology can be achieved only if digital images permit a full diagnostic interpretation. Newer imaging technologies covering the gamut of modalities have taken advantage of the increased speed of computer systems to produce examinations with both more data per image or more images per examination. This trade off between image quality and data amount is particularly true in projection imaging. For example, in angiography, $1024 \times 1024,10$ to 12 bit images are now commonplace, achieving both higher spatial resolution and dynamic range than earlier $512 \times 512$, 8-bit systems.

PACS must be able to handle these large data sets, which can approach 100 megabytes or more per study. Particularly, PACS displays must be able to rapidly recall from memory and paint to displays large images and data sets, and the transmission of images must be efficient to avoid delays in image transfers. The development of an efficient display system required fast computer systems that are now available on the economical PC platform. PACS have become more cost effective partly because of the dramatic cost reductions seen for memory and computational speed.

Similar to the need for faster computers, the large data sets per imaging procedure necessitated an improvement in network bandwidth. Table 1 illustrates the advantage of high bandwidth. The numbers in this Table represent the theoretical minimum transmission time. Although bursts of data can achieve the stated transmission rate, actual transmission times are usually a factor of 5 to 10 slower when communication protocols and other overhead is accounted for. Communication links with fast Ethernet $(100 \mathrm{Mbits} / \mathrm{sec})$ can use the same communication links (twisted pair) and cost no more than standard Ethernet (10 Mbits/sec). The computer cards for ATM and Gigabit Ethernet require fiber connections and are only slightly more expensive than standard Ethernet. ATM will probably by supplanted by Gigabit ethernet for various reasons. Networks also have benefited from the increase in computer speed, which has enabled routers to keep pace with the increased bandwidth.

Another development, which has had the effect of expanding bandwidth, is software for image compression. Both lossless (or bit-preserving) and lossy (or non-bit-preserving) compression is now used extensively in PACS to reduce transmission times and increase storage capacity. Faster computer speeds permit compression or decompression times even for large images to be under 1 second.

\section{Cost of Computer Components}

A remarkable aspect of computer technology has been the increase in computer speed and the reduction in the cost of memory. Cost per megabyte of storage is shown for a number of storage technologies in Table 2 (which most certainly will be obsolete within a year). Storage devices have

Table 2. Digital Storage Devices: Media Cost

\begin{tabular}{lll}
\hline Medium & \multicolumn{1}{c}{ Capacity } & Approximate Cost/Mbyte, 6 \\
\hline CD-ROM & 650 Mbytes & 0.15 \\
DVD & $4.7-17$ Gbytes & $0.85(4.7$ Gbyte $)$ \\
Optical disk & 5.2 Gbytes & 1.4 \\
Magnetic tape & & \\
$\quad(8 \mathrm{~mm})$ & 20 Gbytes & 0.35 \\
RAID & 1000 Gbytes & 10 \\
EIDE PC disk drive & 30 Gbytes & 0.5 \\
Magnetic tape & & \\
$\quad$ (DLT) & 35 Gbytes & 0.2 \\
\hline
\end{tabular}


different benefits and drawbacks, but the price of all have dropped enormously. Excluding $C D$ ROMs, digital tape remains the cheapest, but retrieval times are slower than from optical devices. Both types of storage are used in PACS. Reduced total cost of storage is the key economic element tilting the scale toward PACS archival cost reduction as compared with film. Aside from maintenance, the cost of media for storing a large diagnostic study (eg, $>80$ Mbytes) is negligible in comparison with the cost of the procedure. It should be noted that if such a study were stored on film, not only would the cost of storage be high, but also the ability to view all the images on film would be nearly impossible. For example, an 80 Mbyte MR study would consist of about 600 images and consume 38 sheets of film printed as 16 on 1. Such MR studies, while not universally commonplace, are frequently seen at our institution.

Currently, the cost of storage, computers and network infrastructure have decreased to a point at which the cost effectiveness of this technology can be demonstrated positively for large, high-volume institutions. This is especially so for cross-sectional imaging. The economics of PACS are characterized by higher fixed costs for the digital infrastructure and lower variable or marginal costs related to savings in film and personnel. Because of continuing cost decreases for all forms of digital technology, this trend will go only in one direction, and soon cost-effective solutions will be available for many more hospitals and clinics. Creative use of compression technology will yield further economic benefits.

\section{PACS Workflow}

The last technological improvement needed for a filmless radiology department was a better understanding of workflow within a PACS. That is, a realization that an effective PACS cannot be achieved simply by coupling together hardware. The smooth flow of images to the location at which they are needed, at the time they are needed, and displayed in the preferred manner as required by the radiologist to achieve both throughout efficiency and high diagnostic accuracy, requires management of the studies. This study management, often referred to as folder management, provides the PACS with intelligent functionality for image acquisition, routing, storage, presentation, and retrieval functions. ${ }^{2,3}$ Most processes in the workflow manager are event driven by activities detected in the HIS-RIS. For example, when a patient is scheduled for a given type of procedure, the workflow manager will know where the images are likely to be viewed, what prior studies are relevant and prefetch these studies from the long-term archive and then to the workstation before the arrival of the images from the current study. Reports from prior procedures will be available at the workstation. When the study is completed the images are automatically sent to the PACS and routed to the appropriate workstation. When the new study is called up for review, images appear in the correct sequence and at the appropriate window width and level. Prior images are available immediately for display.

\section{PACS-RIS-HIS INTEGRATION}

Medical imaging is increasingly a key triage event in a patient's encounter with healthcare. Imaging can distinguish between acute and chronic problems and whether the problem being evaluated is surgical (ie, anatomic) or medical (ie, biochemical). The increasing economic stresses on medicine demand efficiency. The evaluation of a patient includes the patient's complaint, past history (preferably documented and retrievable), physical examination findings, laboratory test results, and imaging examination findings. The primary care physician, in concert with any specialists involved in the evaluation, needs prompt return of or access to the products of the imaging procedures, including images and diagnostic reports.

PACS, with secure Internet or Intranet web techniques, enables rapid and simultaneous access to images in different physical locations. We are no longer bound by the single physical film study. Misplaced and lost examinations are significantly less common with PACS. With prompt interpretation by radiologists and voice recognition technology, reports should be available rapidly and automatically associated and delivered with the images. However, successful PACS requires RIS and HIS integration.

The most important roles of HIS related to PACS are to provide a "clean" master patient index (MPI) that identifies uniquely every patient on a one-to-one basis, as well as to provide admission, transfer, and discharge information. The RIS pro- 
vides notification of events-particularly the scheduling of an imaging procedure, with relevant clinical information. The RIS provides unique identification of the imaging procedures requested and performed, allowing the PACS to handle and archive them unambiguously, and to associate a diagnostic report with each study.

Problems may occur even with highly integrated PACS-RIS-HIS. One of the most bothersome is that the PACS database, even with the best prefetching, usually is unaware of prior studies existing only on film. In addition, the RIS database at this time usually does not distinguish between studies on film and those archived digitally, without film. ${ }^{4}$

As shown at the Integrated Healthcare Enterprise (IHE) symposium at 1999 RSNA Meeting (Radiological Society of North America), ${ }^{8}$ userefficient integration requires the ability to maintain focus on a specific patient as the user moves from the PACS, for example, to the HIS or the clinical lab system. The data view presented to the user should be consistent regardless of the source of information. The goal of the IHE effort is to achieve visual integration or clinical context, resulting in the ability to view a specific patient's entire clinical situation. Web founder Tim BernersLee's vision of information management and distribution systems is "The appearance of the information and the tools one uses to access it should be independent of where the information is storedthe concept of location independence. ..." Clinical image and information systems are far from this ideal today.

\section{NEW SOFTWARE TECHNOLOGY}

The future promises many changes in the way information system components are designed, and more importantly, how they interact or "interoperate." Medical information systems in general have evolved as islands, and their interactions today are through protocols and standards such as $\mathrm{HL}^{7}$ and DICOM. ${ }^{10}$ A protocol defines the specifics of communication between the separate systems, whereas the term standard also invokes a set format in which the data are stored. In this sense, HL7 and DICOM are both protocols and standards. In the web example, HTTP (hypertext transfer protocol) is a communications protocol, and HTML (hypertext markup language) is the data standard.

A typical health care enterprise today has as many as 20 independent system databases. In the past, there have been attempts to duplicate the clinical data in a central repository, a clinical information system, through interfaces with protocols and standards like HL7, as well as proprietary interfaces and intermediary computer devices known as "interface engines." Recently, there seems to be a trend toward an acceptance of these distributed patient data systems, with dedicated systems for different departments and functional areas such as admitting, the intensive care units, and the cardiac catheterization laboratory. In this manner, systems are designed specifically for these dedicated areas, and institutions can select the "best of breed" to put together the component systems that meet their needs. In the future, these distributed systems will be solidified through web technology, which makes these separate systems readily accessible through the URL (universal resource locator) concept.

Evolving web technology holds great promise for the future of radiology and medicine. Today's web technology focuses on the presentation or appearance of data through HTML. In the future, XML (Extensible Markup Language) and RDF (Resource Description Framework) will concentrate on the content of data through data description tags that are developed by consensus through standards bodies such as HL7. Then, for example, today's admissions at UCSF between 10 and 11 AM could be accessed by a query process that uses the web by setting up a request to the URL: hospita1.ucsf.edu/admit/10-11. This page would be updated immediately with any new admission. Other database applications could access this page and inspect (parse) it for new admissions. The RDF for this resource would define the set of data descriptor tags so that other database applications could insert the information for these patient admissions into their own databases as if they were doing the same query. In this way, information could be shared among all of the institutions' relevant databases. ${ }^{11}$

Other software technologies such as CORBA (Common Object Request Broker Architecture) $^{12}$ will probably provide a transition for DICOM in the future. CORBA allows objects and their methods on the other computers to be invoked by a given computer. For example, a CT scanner computer may request storage services from an object residing on a server in the PACS computer room using CORBA over an Intranet or the Internet. The 
object broker would replace the current DICOM association (and possibly negotiation) processes.

\section{EMERGING TECHNOLOGIES FOR HEALTH CARE}

The Use of Computers for Data Acquisition, Distribution, and Display

Hand-held devices. The development of handheld devices such as the Palm Pilot is changing the landscape for the interaction between man and the computer. Currently, these devices, called personal digital assistants (PDA), are used primarily for calendars, address and phone books, and to-do lists. Usually these devices have infrared wireless communication to an adjacent PDA or personal computer as well as the more traditional wired interfaces. Now new PDA devices are becoming available that also include an RF interface similar to a cell phone, along with software for handling e-mail and even stock market trading.

Similar applications for health care have been meager at best. The slow speed for communications, hand-written data entry rather than keyboard entry, limited memory, and low-resolution displays have made such developments difficult. The dream of using such devices to interact with a patient's medical record at the bedside is quite attractive. Speech recognition clearly will facilitate success in this arena.

Speech recognition. Speech recognition has finally become a technology useful for radiology. ${ }^{13,14}$ By replacing the entire transcription process, speech recognition promises to dramatically reduce costs while speeding our consultation reports to the referring physicians. Accuracy rates for these devices are now quite acceptable, and these systems are being installed at institutions around the country. There are a couple of potential disadvantages, however. One problem is that it can take longer for the radiologist to generate a report with speech recognition than with conventional dictation. The differences usually are not that great, less than $5 \%$ to $10 \%$, but enough to discourage use in high-volume practices. Savings for the hospital, if the hospital is paying for transcription, cannot be given easily to the radiologists because of federal regulations. If this were the case, the additional effort required by the radiologists could be compensated.

Speech recognition on the hand-held device could make the PDA extremely powerful, elimi- nating the time for and obstacles to data entry. Coupled with an electronic medical record, such devices truly could eliminate most use of paper and all of the problems associated with paper management.

To provide speech recognition on the PDA, a number of new technological developments are needed. Speech recognition hardware and software need to be miniaturized and able to run on the batteries in a PDA. The electronic medical record must be developed more fully. The imaging capabilities of the PDA must improve. The interactions between the various systems need to be much more robust than the current level of HL7, which is the communication standard used by most hospitals among departmental systems. And the RF transmissions to the PDA must become faster to handle large files such as images.

Web applications. The web and E-commerce are changing the way we live. The web grocery store lets us order our groceries for home delivery, keeping track of our favorite items to make selection fast and easy. On the web, one can select the best flight at the best price and then reserve the hotel room at his destination. Examining the car of one's choice with the desired color and options is a few clicks away. But what about health care, especially radiology?

Numerous "dot-com" companies related to health care are springing up (and merging). Most attempt to attract the public, exposing them to advertising, as they explore various health care subjects and options. The thirst for information about health has been the selling point to the venture capitalists who are investing in these new companies. Most of these start-ups are not yet profitable and many never plan to charge the public, living instead on the advertising revenues.

Hospitals and physician groups are creating web pages to attract patients and their health plans in regional markets. These institutions ultimately will allow patients to schedule doctors' visits, seek advice about common diseases, learn about new techniques and facilities, and check on their own medical records. The patients will be exposed to information about good nutrition and the virtues of avoiding smoking, drugs, or excessive drinking.

For the radiologist, specific web applications have been limited to teleradiology (remote access to PACS), digital teaching files, and limited numbers of Continuing Medical Education (CME) 
courses. The web has enabled the radiologist to gain access to reference materials and communicate via e-mail with referring colleagues. Policy and procedure manuals, protocols, patient preparation information, and work schedules all are available on the web. The web is rapidly becoming the platform for the RIS and for the integration with the HIS and electronic medical record (EMR) to be described below.

Integration of the Healthcare Enterprise. The concepts of the Integration of the Healthcare Enterprise (IHE) are vital for the aforementioned interactions to be achieved. ${ }^{8}$ The IHE program will bring the various hospital information system vendors together with the PACS vendors to move beyond the DICOM standard for image exchange. Through the IHE effort, laboratory data, allergy history, rationale for ordering the imaging examination, and pertinent clinical history will be presented to the radiologist while he is viewing images for interpretation.

In the IHE, the patient's data, although probably residing on multiple independent distributed systems, appears as if in one virtual database. Decision support tools can then extract information from this database to guide the clinician in the diagnostic workup as well as in the therapeutic decisions. This type of information is patient-centric, which differs from extraction of data for research or teaching purposes.

\section{The Use of Computers for Patient Management}

Electronic medical record. Physicians will use the web and Internet to access their patients' electronic medical record (EMR) from home or while away from their primary office. They will be using the web in their office or hospital as well. In fact, the web likely will be the primary means with which they access the EMR, and through which they will communicate with other physicians and health care providers as well as with their patients.

The implementation of the EMR has been hampered by the lack of effective speech recognition for physician input and the difficulty in establishing precision in medical terminology. The "art" of medicine so far has precluded widespread use of standard terminology, which is required to put enough structure into the medical lexicon to create the robust databases needed for the EMR. One example of a successful implementation of standards in medical terminology is mammography.
Decision support applications. The secret to the effectiveness of decision support applications is to provide the specific subset of relevant data and only that data the physician needs at any point. For this approach to work, the system must understand what information the physician already has seen and to remind the physician about important information that may have been forgotten. Clinical protocols should guide the physician through the decision-making process, suggesting reasonable alternatives with a guide to advantages and disadvantages of each option. These sidebar interactions must be activated only if the physician so desires, so as not to distract or divert attention and time unnecessarily.

Extraction of data from a large database often is described as data mining. Examples of such data mining would be extracting images for research and teaching purposes based on radiologic findings and anatomic location. Linkages between a current case under review and similar cases based on findings or clinical setting would be a powerful tool for either differential diagnosis of a tough case or teaching the residents. As was shown by Dr Swett years ago, series of images could be presented that are related based on different presentations for the same disease, different diseases with the same presentation, and different presentations based on severity or on duration of the disease. ${ }^{15}$ All of these require a robust database and powerful retrieval capabilities, with complex data relationships, searching, and sorting.

Outcomes analysis. Outcomes research will become far more important in health care in general and in radiology in particular. Well-designed and well-organized outcomes analyses need to be performed to look at the cost effectiveness of imaging studies. This technology assessment is critical to the future of radiology particularly with stiff competition for health care dollars and capital budget provisions. The ability of the radiologist to become the "informaticist" will depend, to a large degree, on the quality of outcomes data that can be accumulated and available. Decision-support tools must have built-in references to solid outcomes data.

Outcomes data are already being used to give physicians and health care institutions report cards supposedly to provide the public with information about the quality of care. These outcomes data also will be the basis for algorithms in the decision 
support processes to provide probabilities for diagnostic and therapeutic alternatives. These data will guide the referring physicians in the proper selection of studies.

Such outcomes data can be used to monitor drug effectiveness and interactions in large populations, follow altemative treatments, evaluate cost effectiveness of screening programs, rank the utility of diagnostic tests, and provide accurate measurements of physician and hospital performance. For these processes to be useful, the quality and quantity of such data has to be improved. Data entry into these sophisticated databases requires a robust EMR.

Teleradiology and telemedicine. Teleradiology will provide the radiologist with the capability to cover community and rural institutions from the comfort of their main office without having to travel across town. ${ }^{16}$ Military hospitals as well as prisons can be covered in this manner. Subspecialty-trained radiologists will be able to consult on tough cases or provide direct primary interpretations. TeleQuest, a national consortium of academic institutions, is one commercial venture providing such services. Second opinions by subspecialty experts likely will increase dramatically, especially when report cards are published for each practicing radiologist. Internationally, the use of teleradiology will provide better coverage remotely, as well as create new opportunities for academic radiologists in this country.

While on call from home or from a remote office, radiologists will use the web to access the patients' EMR, images, and radiology reports. The connectivity made possible by IHE will make these interactions seamless and efficient. When the radiologist is presented with a new examination, the pertinent clinical history, laboratory findings, and operative notes will be summarized appropriately along with the previous reports and images. The decision support tools will guide the radiologist through the interpretation process with weighted differential diagnoses based on data in the patient's total EMR.

Computer-aided diagnosis. Computer-aided diagnosis (CAD) tools will be very important as an assistant to the radiologist. CAD can draw attention to findings on images, including highlighting changes from previous images. CAD currently is in use for the identification of microcalcifications and masses in mammography. Already, the ability for these systems to detect possible malignant tumors is reported to surpass that of many radiologists and to now equal or almost equal the detection skills of the most advanced mammographer. ${ }^{17}$ A large number of false-positive results still limits its utility. Yet, having the system call attention to possible abnormalities is as good as, if not better than, having a second reading. Second readings have been shown to improve detection, simply because another set of experienced eyes are viewing the same images. In the future, similar techniques could make screening $\mathrm{CT}$ for coronary artery calcification, lung cancer, and other common conditions cost effective. Certainly, such aids could help reduce the variability in detection of abnormalities on the part of all radiologists.

Virtual imaging and 3-dimensional presentation. Virtual CT colonography already has proven to be an effective method of examining the colon to rule out cancer. ${ }^{18}$ Currently, a large amount of time is spent by the radiologist and by technicians to create the optimized 3-dimensional images for interpretation and presentation. As the computer techniques improve, this use of CT might become far more cost effective and less invasive than colonoscopy.

Three-dimensional (3D) image presentation for a variety of imaging procedures will become more important for both the radiologist and the referring physicians in the years to come. The very large image sets created by multislice CT, MR, interventional procedures, and ultrasound require an efficient process for viewing the images. Currently, 3D presentations are limited to CT, MRA, and virtual colonography, with neurosurgeons routinely using 3D presentations to augment their surgical approach with superimposed wands or other instruments. 3D presentations coupled with multimodality overlays and CAD will add more dimensions to the radiologist's perspective. For instance, the beating heart will be projected not only with surface rendering to see the coronary arteries but with time-dependent views of contrast enhancement including transparent views to see the endothelium and both hard and soft plaques.

The 3D image set for breast MR will be integrated with the MR spectroscopy data as well as a set of ultrasound images with speckle-corrected data created by mild compression of the breast under examination. The areas of possible abnormality will be highlighted with graphic presentations of the statistical likelihood of benign versus malignant disease. 
Guidance and visualization systems. Interventional radiology will be enhanced by MR interventional techniques such as automated steerage of microinstruments on the tips of very fine catheters. ${ }^{19}$ These "intelligent" catheters will be guided into extremely small vessels under computer and joy-stick control. Once at a target location, the microcircuitry at the tip will be activated, performing a biopsy or depositing an implant or genetically altered cells. Of course, these catheters also will be able to take an image with either optical imaging, ultrasound, or serve as transmitting or receiving $\mathrm{RF}$ coils for MR. Hybrid rooms, such as the one under construction at UCSF, will combine fluoroscopic interventional techniques with high-field MR. Reaching a very small berry aneurysm deep in the brain will be facilitated by such techniques, further reducing the morbidity and mortality associated with these conditions.

\section{MEDICAL IMAGING IN THE NEXT DECADE}

\section{Changes in the World of Radiology}

Radiology is "going digital" and will increasingly be the key triage event in a patient's encounter with health care. More and more, CT and MR are being used for screening such as in the evaluation for coronary artery calcification and lung cancer. PACS will continue to be refined and gradually replace film as an image management system. Multislice CT and large MRA studies are now becoming commonplace and difficult as well as expensive to manage and view on film.

Enhancements to the folder manager will allow for image processing techniques to be applied to images based on history, previous results, or the questions being asked. Radiologists will continue to be invaluable for consultation, differential diagnosis, examination protocoling, and examination management. A big challenge for the radiologist will be that of contemporaneous interpretation. If radiology meets this cultural challenge for change, then the radiologist of the future likely will be much more involved in the diagnostic pathway than is now the case. If not, radiologists could become irrelevant when images are released to the clinical environment.

\section{Radiologist as Health Care Informaticist}

Currently, the primary care physician often manages the patient through the complex diagnostic

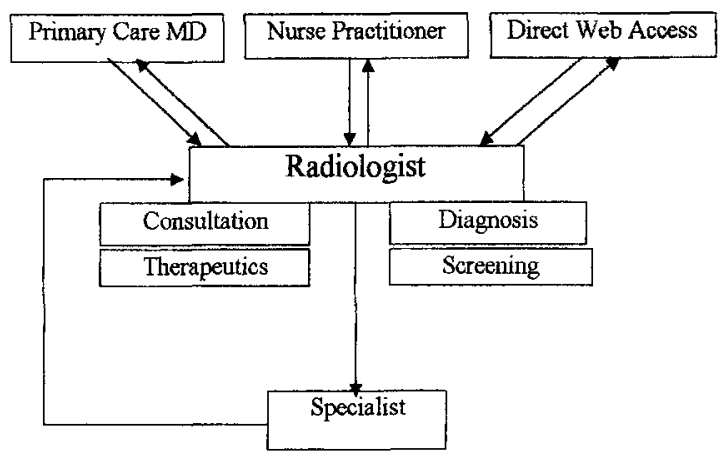

Fig 1. Future role of the radiologist/informaticist.

workup as well as decides when and with whom to consult for the care of the patient. With the expanding role that imaging plays in the diagnostic workup and therapeutic pathway for our patients, the radiologist could become much more directly involved in managing the patient through the health care system and, in the process, replace a fundamental role of the primary care physician. Figure 1 shows a simple schematic representation of this new role for the radiologist. Patients will continue to see primary care physicians or, increasingly, nurse practitioners. But a third entry point will be the patient directly seeking care, often through the Internet and the web. All 3 of these pathways will lead to the radiologist, especially when there is any doubt about the proper diagnostic pathway or selection of specialist, if needed.

The radiologist would then be in the position to decide on the appropriate diagnostic tests and then, based on the diagnostic tests as well as history and physical findings, the best therapeutic approach including the correct specialist for the patient. Obviously, this process can be successful only if the radiologist has the appropriate information available in a concise and convenient format. The tools described above, such as decision support, outcomes analysis, and CAD are essential for this approach to work.

One scenario that illustrates this new role would be a teenage patient with a first-time seizure. This patient would call the nurse practitioner or communicate via the web and would immediately be referred to the radiologist. The radiologist would review a summary of the EMR for that patient, which was either entered by the patient directly or from the nurse practitioner. The summary was created by the decision support applications in- 
cluding the protocols for seizures. In this relatively simple example, the radiologist's personal involvement would be quite limited, and the patient would be scheduled for the brain MR. The neuroradiologist interprets the scan and finds a small focus of abnormal tissue on the echo-planar sequences. The triage radiologist, in consultation with the neuroradiologist, then sends the patient to the interventional neuroradiologist for treatment. This process would be documented in the EMR, and the nurse practitioner as well as the patient would have access to these records.

To understand the use of the PDA and this new role for the radiologist on the in-patient side, another scenario would be useful. Imagine the referring physician visiting her patient at the bedside. After reviewing the results from the laboratory (data from the HIS) and last night's chest film (data from the PACS) on the PDA, she could order the chest $\mathrm{CT}$ (connecting with the RIS) and send a consult request to the pulmonary specialist. The CT order interaction ensures the proper ICD9 codes. The referring physician, when requesting the chest $\mathrm{CT}$, would be prompted to enter the history and reason for the CT. The system then would provide the correct ICD9 code. Through a streamlined optimization strategy, the request could be changed to a more relevant magnetic resonance angiogram (MRA) of the thorax, based on the history and chest film findings. The physician would be guided to the MRA through a series of brief questions refining the nature of the problem to be solved. If the proper diagnostic imaging workup findings are ambiguous, the referring physician would be connected to a consulting radiologist for help.

An alert system associated with this PDA could notify the referring physician that new laboratory results on another patient in the hospital are available, and the important new information, requiring prompt attention, could be shown on the display device. When the chest CT and report are available, the alert is sounded, and the physician can view selected images (chosen by the radiologist) on the PDA in small image format. Of course, the radiology consultation report generated by speech recognition also is available, as well as digitized speech of the report impression.

Hopefully, many of the interactive consultation tools discussed here will become a reality. Radiology must embrace the new technology and change to accommodate it into our workflow, or risk becoming a dinosaur. If we become the early adoptors of these technologies, the radiologist will become the health care "informaticist" of the future.

Failure to perform prompt interpretations or become directly involved in the real-time assessment of patients will make it possible for other specialists to do more imaging interpretation as well as maintain the role of managing the patient. The use of image and information management technologies can pull the clinician and the radiologist closer. Good interpersonal skills and customer relations are more important than ever as well as the real-time service orientation.

\section{SUMMARY}

These emerging technologies and the changing environment in radiology are going to force the radiologist to change the very nature of his practice. One possible approach that would enhance the role of the radiologist would be to become the health care informaticist and to become more directly involved in the triage and management decisions for the patient. This role would demand not only prompt, real-time image interpretation, but also use of the informatics tools such as decision support, outcomes analysis, CAD, and virtual imaging. The radiologist will need to be trained and prepared to manage the decision process for the patient, taking full advantage of the new PDA, web applications for the EMR enhanced by the IHE, and teleradiology and telemedicine. Failure to take this bold approach could leave the radiologist more vulnerable to lose imaging as well as end up in a marginal role in the future management of patients and the health care delivery system.

\section{REFERENCES}

1. Arenson RL, Avrin DE, Wong A, et al: Second Generation Folder Manager for PACS. Proceedings of SCAR ' 94. Winston-Salem, NC, June 12-15, pp 601-605, 1994

2. Seshadri SB, Kishore S, Arenson RL: Software suite for image archiving and retrieval. Radiographics 12:357-363, 1992
3. Arenson RL, Seshadri SB, Stevens FJ, et al: The overlapping domains and interface between radiology information systems and medical image management systems (PACS), in Lemke HU, Rhodes ML, Jaffee CC, et al, (eds): Proceedings of Computer Assisted Radiology. Berlin, Springer-Verlag, 1987, pp 855-865 
4. Andriole KP, Avrin DE, Yin L, et al: PACS databases and enrichment of the folder manager concept. J Digit Imaging 13:3-12, 2000

5. Andriole KP: Computer and digital radiography, in Hangiandreou NJ, Young JWR, Morin RL (eds): Electronic Radiology Practice-Technical and Practical. Oakbrook, IL, 1999, pp 35-41

6. American College of Radiology, National Electronic Manufacturers Association: ACR-NEMA Digital Imaging and Communications Standard, NEMA Standards Publication No. 300-1985, Washington, DC, 1985

7. http://www.hl7.org/

8. http://www.rsna.org/IHE

9. Berners-Lee T with Fischetti M: Weaving the Web. San Francisco, CA, Harper, 1999, p 117 and 159

10. Digital Imaging and Communications in Medicine (DICOM) Standard. NEMA. 2102 L Street NW, Washington, DC. http://www.nema.org/

11. Bosak J, Bray T: XML and the second generation web. Sci Am May, 89-93, 1999 http://www.xml.org

12. http://www.corbamed.org

13. Ramaswamy MR, Chaljub G, Esch O, et al: Continuous speech recognition in MR imaging reporting: Advantages, disadvantages, and impact. AJR, 174:617-622, 2000

14. Rosenthal DI, Chew FS, Dupuy DE, et al: Computerbased speech recognition as a replacement for medical transcription. AJR, 170:23-25, 1998

15. Mutalik P, Fisher P, Swett H; Radiologic knowledge representation \& axis display in the IMAGE/ICON System, in Arenson RL, Friedenberg RM (eds): SCAR '90, Philadelphia, PA, Symposia Foundation, 1990, pp 185-190

16. Boland GWL: Teleradiology: Another revolution in radiology? Clin Radiol 52:547-553, 1999

17. Memdez AJ, Tahoces PG, Lado MJ, et al: Computeraided diagnosis: Automatic detection of malignant masses in digitized mammograms. Med Physics, 25:957-964, 1998

18. Fenlon HM, Nunes DP, Schroy PC 3rd, et al: A comparison of virtual and conventional colonoscopy for the detection of colorectal polyps. N Engl J Med 341:14961503,1999

19. Roberts TPL, Hassenzahl W, Arenson RL: Proceedings of the 8th Annual Meeting of the International Society for Magnetic Resonance in Medicine, program \#405, Denver, CO, April 2000 